\title{
Displacement maximisation of haunched-rafter Pitched-roof Steel Portal Frames
}

\author{
H. K. Issa \& F. A. Mohammad \\ School of Architecture, Design and the Built Environment, \\ Nottingham Trent University, UK
}

\begin{abstract}
Pitched-roof Steel Portal Frames (SPFs) are common structures used in single storey buildings. It is necessary for this popular steelwork to pass through an optimisation process to minimise the total cost of the frame. Optimisation in terms of weight is well documented with different optimisation techniques. However, this approach to displacement maximisation is somehow rare. In this paper, an attempt is made to perform optimisation in terms of lateral displacements. To achieve this, a modified distributed genetic algorithm (DGA) is used to maximise lateral displacements of the SPF while the constraints meet the requirements of BS 5950. Furthermore, software 'DO-DGA' (Design Optimisation using DGA), coded by Visual Basic 6.0, has been developed by the authors to loop the optimisation process. Although the appearance and form of SPFs are simple, according to BS 5950, there are more limitations to be checked than in complex structures. A stiffness matrix has been derived for the haunched part of the rafter using a column analogy and a virtual work method to involve this part in the analysis process. Through two benchmark examples, the comparison is made between the results of weight minimisation and displacement maximisation.
\end{abstract}

Keywords: distributed genetic algorithm, steel portal frame, optimisation, displacement maximisation.

\section{Introduction}

Single storey buildings are widely used in the UK; it is estimated that $50 \%$ of the single storey steel work buildings are constructed by steel portal frames [12]. Because of its economy and versatility for large spans in the construction of 
pitched roofs such as shopping centres, warehouses, retail shops, pools, factories, etc, the steel portal frame has become the most often used structure within this sector.

Any structural designer attempts to conduct an economical design. This can be achieved by formulating a design problem and solving it by an optimisation technique while meeting the requirements of a code of practice to control the safety of the structure [13]. However, due to the large number of iterations in implementing the optimisation technique, it cannot be achieved by using the designer's experiences and intuition. As it is believed that the major cost of structural steelwork is its own weight, approaches to minimising the weight have become increasingly interesting for researchers. However, it necessitates choosing different approaches for those types of structure that are controlled by deflection and displacement. Minimising the weight is relatively well documented in literatures (see, for example, $[1,4,6,7,10,13]$. To the knowledge of the authors there has not been any attempt to investigate the displacement maximisation of the steel structure thus far. In this paper, a modified distributed genetic algorithm (DGA) is implemented to conduct both weight minimisation and displacement maximisation. Through two benchmark examples, the comparison is made to portray the more effective approach for the design optimisation.

\section{Distributed genetic algorithm}

The basic mechanics of the Genetic Algorithm (GA) are based on randomised procedures of the selection and reproduction of the population of individuals and copying the fittest individuals into the next generation. A GA moves from one generation to another until either a certain individual dominated population or a predetermined maximum number of generations are reached. A basic GA consists of three main operators; reproduction, crossover and mutation. In the reproduction stage, a set of the population are selected for mating depending on their fitness values, which represent the objective function. If any constraint is violated, a penalty is applied to the objective function. The value of the penalty is related to the degree to which the constraints are violated.

Camp et al. [2] used three crossover schemes, fixed, flexible, and uniform, to minimise the weight of the structure. The crossover and mutation probabilities were 0.85 and 0.05 and the maximum crossover point was 3 . Kameshki and Saka [6] applied a GA for the optimum design of unbraced multi-storey frames with semi-rigid beam-to-column connection. They adopted constant values of 0.001 for mutation probability. Toropov and Mahfouz [13] modified the GA to improve its rate of convergence. The modified GA was linked to a system of structural design rules, interacting with a finite element package in order to obtain minimum weight designs of plane structural steel frames. Saka [11] studied the optimum design of pitched roof steel portal frames using the GA. He used only gravity loads to minimise the weight of the steel portal frame. Degertekin et al. [3] implemented the GA to investigate the optimal load and resistance factor design. They applied a uniform crossover and a simple mutation 
probability with values of 0.001 and 0.002 , as they found it suitable in the examples. The elapsed time for the process was $21.2 \mathrm{~min}$ while 4512 frame analysis was required (6 design variables).

In the DGA, the performance of the conventional GA is improved by some minor modifications in its main algorithm that leads to quicker convergence and higher searching capability compared to the conventional GA [9]. Adopting the migration idea of the population, the DGA uses a number of population groups and implements genetic operations in parallel for all populations existing in different groups. Then, the best populations of each group migrate to another group, making it possible for them to contribute in quicker converge than the GA into an optimum solution.

In this research, a DGA has been modified for the purpose of improving the algorithm performance and saving the computation time in convergence into an optimum solution. Furthermore, an attempt is made to express the design problems in terms of weight minimisation and displacement maximisation to articulate and highlight the efficiency of both objective functions. For this purpose a software coded by Visual Basic 6.0, called Design Optimisation with DGA (DO-DGA), has been developed to conduct the optimisation process. The major aspects of modification that DO-DGA follows are stated as the following:

- In addition to other crossover schemes defined by literature review, a four-point crossover has been added to the crossover scheme.

- Although twin is not meaningful in genetic algorithm, DO-DGA had adopted this idea to produce more offspring. As the best parents in the population can give better offspring, a probability has been assigned to the parents that allows them to undergo crossover operation twice, resulting in producing more offspring. This will make it possible to increase the number of better individuals among the population of the group.

- The elite individuals are included in genetic operations while they are already secured to be dropped into the next generation. The elite individuals are participating in reproduction twice to increase the existence of fit individuals among the population.

- Similar individuals in the population do not undergo structural analysis and fitness calculation as many times as they are. Because of similarity in the population, the process is conducted once and the fitness value is assigned to all similar individuals at the same time.

- In contrast to the literatures which have addressed a constant value for mutation probability, DO-DGA uses a varied mutation probability. This assists the algorithms to make more diversity among population and consequently more feasible design spaces can be employed to reach the fittest individuals. Reaching to global optimum requires best diversity among population. The mutation probability can be formulated as follows:

$$
P_{m}^{G_{C}}=P_{m}^{\max }-\frac{e^{-1 / 20}-e^{-G_{C} / 20}}{e^{-1 / 20}-e^{-N_{G} / 20}}\left(P_{m}^{\max }-P_{m}^{\min }\right)
$$


where $P_{m}{ }^{G c}=$ mutation probability of the current generation; $P_{m}{ }^{\max }$ and $P_{m}{ }^{m i n}=$ maximum and minimum mutation probabilities; $G_{C}=$ number of current generation; and $\mathrm{N}_{\mathrm{G}}=$ number of predetermined generations.

- The algorithm uses a DO-DGA penalty function when the aim is to minimise the weight as below:

$$
C= \begin{cases}0 & \text { If } \mathrm{g}_{\mathrm{i}} \leq 0 \\ g_{i} & \text { If } 0<\mathrm{g}_{\mathrm{i}} \leq 1 \\ 2 g_{i} & \text { If } \mathrm{g}_{\mathrm{i}}>1\end{cases}
$$

Whereby the fitness function is defined as follows:

$$
F=W(1+C)
$$

Whereas a simple yet 'exact' penalty function [13] is adopted for maximising the lateral displacement.

$$
F=\left\{\begin{array}{cc}
\sum \delta u & \text { If } g_{\mathrm{i}} \leq 0 \\
0 & \text { If } \mathrm{g}_{\mathrm{i}}>0
\end{array}\right.
$$

where, $g_{i}=$ value of constraint $i ; F=$ fitness value; $W=$ total weight of frame; $\sum \delta u=$ sum of lateral displacement; and $C=$ penalty value.

In design optimisation using genetic algorithm, the individuals that violate the constraints are not commonly discarded as it is believed that those individuals may produce better offspring after crossover and mutation operations. However, it is necessary to discard them when the aim is to maximise the lateral displacement. This is because the fitness function, in this case, depends not only on area and length (volume) but also on moment of inertia (stiffness). As there is no one-to-one relationship between the area and the moment of inertia of the section, formulating a comprehensive fitness equation is somehow impossible, because the bigger area for the section does not imply to have bigger moment of inertia and vice versa. As a result, lateral displacement of the bigger area may be larger than the lateral displacement of smaller area.

In addition to those modifications, DO-DGA uses a general stiffness matrix for both prismatic and non-prismatic members which will be explained later.

\section{Analysis}

As long as the direct stiffness method is used to analyse the structure, it is required to form stiffness matrices for structural elements and add them up to reach a global stiffness matrix for whole structure. The stiffness matrix for prismatic member is well documented through the text books. On the other hand the derived stiffness matrices for non-prismatic members are time-consuming as a subroutine must be formed to calculate the integrals' equations of the matrix elements. Saka [11] implemented the stiffness matrix of non-prismatic member developed by Matheson et al. [cited in 11] to carry out the optimization process on steel portal frames. Luo et al. [8] adopted the transfer matrix method to a deduced general expression for the components of the stiffness matrix of nonprismatic members. Both stiffness matrices have been formed by some 
integration components. In this paper a virtual work method and a column analogy [5] is used to derive a general stiffness matrix for the structural elements. The virtual work was implemented to derive the axial stiffness coefficient, whereas a column analogy was employed to derive the non-prismatic stiffness matrix for bending and shear effects. Accordingly, a member's stiffness matrix may have the form below:

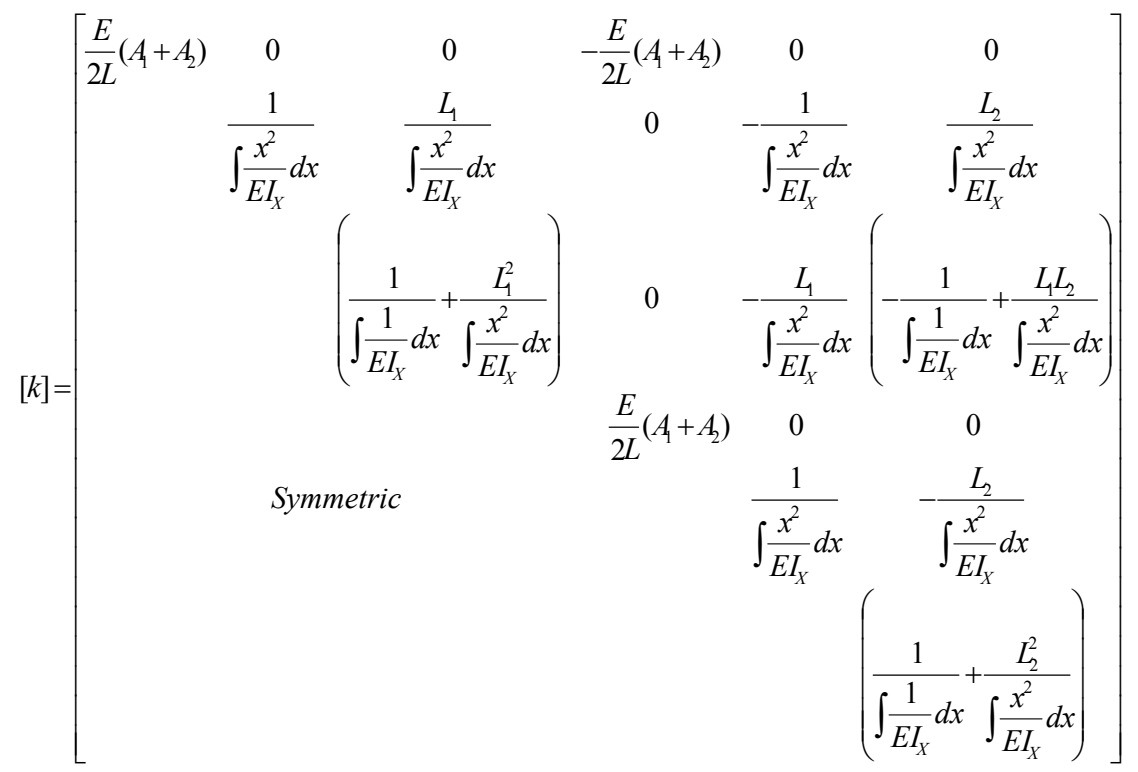

where $k=$ stiffness matrix of single member; $E=$ modulus of elasticity; $I_{x}=$ varied moment of inertia; $L_{1}$ and $L_{2}=$ distances from member ends to the elastic centre; $A_{1}$ and $A_{2}=$ areas of member ends.

To eliminate the integrations in matrix elements, the member's stiffness matrix is passed through regression analysis whereby an additional subroutine is not required to code for the integration. Entire eighty standard steel sections available in BS 5950 are engaged into regression analysis. After immense regression analysis and data collection the stiffness matrix can be refined as below.

When $\mathrm{A} 1 \geq \mathrm{A} 2$

$$
[k]=\frac{E}{L^{2}}\left[\begin{array}{cccccc}
0.50 A^{\prime} L & 0 & 0 & -0.50 A^{\prime} L & 0 & 0 \\
& 0.32\left(12 I^{\prime} / L\right) & 0.36\left(6 I^{\prime}\right) & 0 & -0.32\left(12 I^{\prime} / L\right) & 0.29\left(6 I^{\prime}\right) \\
& & 0.39\left(4 I^{\prime} L\right) & 0 & -0.36\left(6 I^{\prime}\right) & 0.28\left(2 I^{\prime} L\right) \\
& & & 0.50\left(A^{\prime} L\right) & 0 & 0 \\
& & & & 0.32\left(12 I^{\prime} / L\right) & -0.29\left(6 I^{\prime}\right) \\
& & & & & 0.29\left(4 I^{\prime} L\right)
\end{array}\right]
$$


where, $I^{\prime}=$ sum of moments of inertia of member ends; $A^{\prime}=$ sum of areas of member ends; $L=$ length of the member.

For the prismatic member where the difference between the depths of member ends is zero, the stiffness matrix will have the form of conventional one given in any structure analysis textbooks.

\section{Optimum design to BS 5950}

BS 5950 states that when an elastic analysis is used for the design of steel framework such as the one shown in fig. 1, the capacity and buckling resistance should be calculated. It is required to use the effective length equal to that between two intermediate restraints.

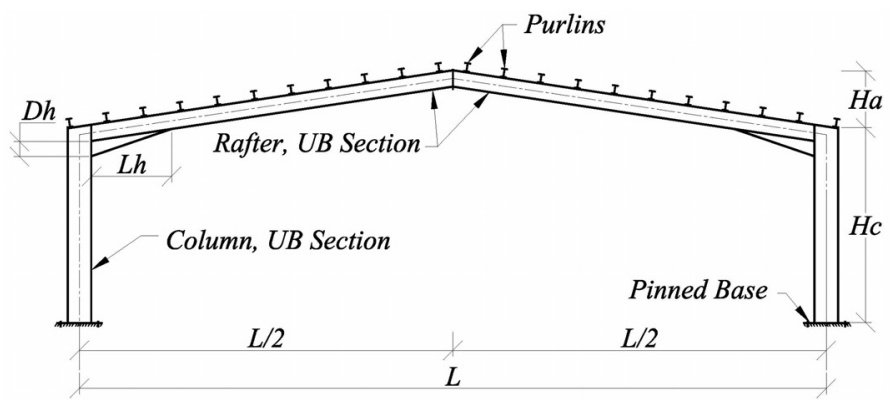

Figure 1: $\quad$ Typical pitched roof steel portal frame.

In the design of pitched roof steel portal frames, it is common to have the same universal beam section for both rafters and a different universal beam sections for the columns. For the reason of economy, the same section of rafter is used to produce the haunch. Therefore, the optimum design of the pitched roof steel portal frame necessitates using two design variables; one for rafter and its haunch and another for the columns. However, if it is necessary to use different section for the haunched section, the number of design variables increases to three.

The design of pitched roof steel portal frame with haunched eaves, when the objective is obtaining minimum weight and maximum displacement, and the constraints are implemented according to BS 5950 has the following form of formula:

$$
\begin{gathered}
\text { Minimise } W=\sum_{i=1}^{n g} \gamma_{i m} \sum_{j=1}^{n m} V_{j} \\
\text { Maximise } \partial=\sum_{i=1}^{n j} \partial_{i u}
\end{gathered}
$$

Subjected to:

$$
\delta_{i} \leq \delta_{i u} \quad i=1,2,3 \ldots, n j
$$




$$
\begin{array}{rr}
M_{x j} \leq M_{c x j} \quad j=1,2, \ldots, n m \\
\frac{F_{j}}{A_{g j} p_{y}}+\frac{M_{x j}}{M_{c x j}} \leq 1 & j=1, \ldots, n m \\
\frac{F_{j}}{A_{g j} p_{c j}}+\frac{m_{j} M_{x j}}{M_{b j}} \leq 1 j=1, \ldots, n m \\
\frac{F_{j}}{A_{j}}+\frac{M_{j}}{S_{x j}} \leq p_{b} \quad j=1,2, \ldots, n h
\end{array}
$$

where $W=$ total weight if frame; $n g=$ number of member groups; $\gamma_{m}=$ unit weigh of the member group; $n m=$ number of members in a group; $V_{j}=$ volume of member $\mathrm{j} ; \partial=$ total lateral displacements of joints; $n j=$ total number of joints; $\partial_{i u}=$ lateral displacement of joint $i ; \delta_{i}=$ horizontal and vertical displacements of joint $i, \delta_{i u}=$ upper limit of displacements; $F_{j}=$ axial member force of member $j$; $A_{g j}=$ gross cross-sectional area of member $j ; p_{y}=$ design strength; $M_{x j}=$ bending moment about major axis; $M_{c x j}=$ bending moment capacity of member $j ; p_{c j}=$ compressive strength of member $j ; m_{j}=$ equivalent factor; $M_{b j}=$ lateral torsional buckling resistance moment; $S_{x j}=$ plastic section modulus; $p_{b}=$ bending strength.

Eqn. (9) checks the displacement of the joints. BS 5950 has limited the horizontal displacement of the joints to column/300 and the upper limit of the beam deflection is span/360. Eqn. (10) checks the moment capacity of the member section-section. Eqn. (11) defines the load capacity check for beamcolumn with semi-compact or slender cross section. Eqn. (12) is the simplified approach of the overall buckling check for beam-column. Eqn. (13) should be checked at any point of the haunched rafter.

As the nature of the structural optimisation variables are discrete, the solution of the optimum design problem given in eqns. (7) and (8) necessitates selecting universal beam section from the table of standard section for rafters, columns and haunched section.

\section{Benchmark examples}

Two examples are used to illustrate the efficiency of both defined objective functions. The steel portal frames given as examples, are assumed to experience different loads combinations. In addition to the imposed load, one of the frames is subjected to wind load which act only on one of the rafter. Unlike what was done by Saka [11], the structural members are not subdivided into smaller elements whereas the structure is summarised into six members. Furthermore, it is decided to apply seven genes for each design variables to include all eighty standard steel sections into calculation. The variables that exceed from the upper limit of steel section number (80) are altered to a random number between 0 and 79. The steel grade of the sections is S275 and 3 lateral restraints have been used for columns. The length and depth of the haunched part of the rafter are varied to find the optimum size of the haunched member. The length of the haunched part varies between $0.05 \mathrm{~m}$ and 6.40 increasing by $0.05 \mathrm{~m}$ whereas the depth of the haunched part varies between $0.01 \mathrm{~m}$ and $1.28 \mathrm{~m}$ increasing by $0.01 \mathrm{~m}$. As a result, 
the number of point loads is changed on the haunched part and the rest of the rafter. DO-DGA has handled this changing by adding a subroutine for load transfer. The number of population for each group is 26 . This was decided after a number of trials that was done to choose the minimum number which gives the best solution. The maximum number of generations is 100 which the algorithm terminates in a certain generation due to domination of a specific individual. A 0.85 value is adopted for the crossover probability. The maximum mutation probability is 0.20 and the minimum value is $0.0005 .30 \%$ of the population are secured as elite for the next generation. Simultaneously, $30 \%$ of the fit population are chosen to migrate to the other group with the migration interval of 3 generations. A 0.40 value is assigned for the probability of twice crossover to produce double offspring (twins). As there is no guarantee that the GA can give the optimum solution, the program is performed 10 times, represented as the number of the 'seed'. At the beginning the decided number of run was 20 . However, as the optimum result gained within 20 runs was the same as 10 runs, therefore, the number of runs was fixed to 10 for reducing the computation time.

\subsection{Frame with self-weight and imposed load}

\subsubsection{Weight minimisation}

An imposed load of $5 \mathrm{kN}$, in addition to the frame's self-weight, is applied to the pitched roof steel portal frame with the span of $20.0 \mathrm{~m}$, the column height of $5.0 \mathrm{~m}$ and the overall height of $6.5 \mathrm{~m}$ (as shown in fig 2). To design the frame, two groups of population are employed. The best individuals of the first group migrate to the first group to enhance the quality of the first group population. Both length and depth of the haunched part are varied according to the decided range.

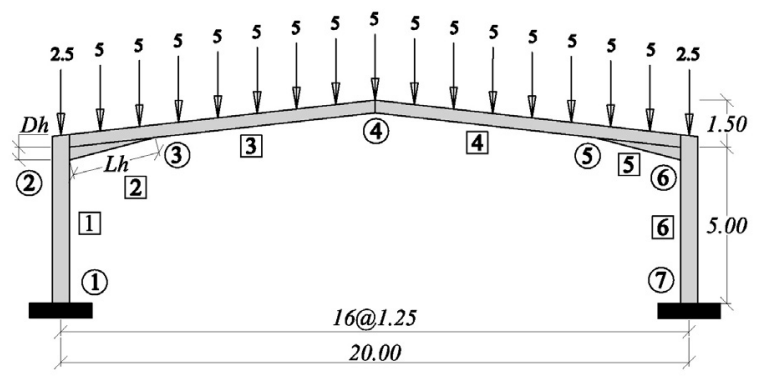

Notes:

All dimensions and loads are in ' $m$ ' and ' $k N$ ' successively.

Joint

Member

Figure 2: The pitched-roof SPF subject to imposed load and self-weight.

The optimum solutions gained after ten runs are given in table 1 .

Browsing the results, the consistency of selecting the steel sections for rafters and columns is remarkable. As the range of the depth and length are quite big 
with smaller step, DO-DGA has found different haunch length and depth at each run. The weights of the optimum solution for ten runs are close to each other showing the efficiency of the program. The optimum gained weight is $1133.9 \mathrm{~kg}$ as shown in runs 3, 7, and 9. The optimum haunch length and depth are $1.60 \mathrm{~m}$ and $0.64 \mathrm{~m}$ respectively. The optimum design was achieved within 43 generations which last 194 seconds. The constraint associated to interaction of the axial force and the moment reached to 0.94 whereas the vertical displacement of the apex reached to its maximum value $(55.5 \mathrm{~mm})$ due to large value of live load.

Table 1: $\quad$ Result of weight minimisation.

\begin{tabular}{|c|c|c|c|c|c|c|}
\hline Run & $\begin{array}{c}\text { Column } \\
\text { section }\end{array}$ & $\begin{array}{c}\text { Rafter } \\
\text { section }\end{array}$ & $\begin{array}{c}\text { Haunch } \\
\text { length }\end{array}$ & $\begin{array}{c}\text { Haunch } \\
\text { depth }\end{array}$ & $\begin{array}{c}\text { Weight } \\
, \mathrm{kg}\end{array}$ & $\begin{array}{c}\text { Gene- } \\
\text { ration }\end{array}$ \\
\hline 1 & $406 \times 140 \times 39$ & $356 \times 127 \times 33$ & 1.60 & 0.69 & 1137.7 & 46 \\
\hline 2 & $406 \times 140 \times 39$ & $356 \times 127 \times 33$ & 1.60 & 0.67 & 1136.2 & 46 \\
\hline 3 & $406 \times 140 \times 39$ & $356 \times 127 \times 33$ & 1.60 & 0.64 & 1133.9 & 49 \\
\hline 4 & $406 \times 140 \times 39$ & $356 \times 127 \times 33$ & 1.75 & 0.58 & 1135.9 & 47 \\
\hline 5 & $406 \times 140 \times 39$ & $356 \times 127 \times 33$ & 1.65 & 0.65 & 1137.0 & 55 \\
\hline 6 & $406 \times 140 \times 39$ & $356 \times 127 \times 33$ & 1.75 & 0.58 & 1135.9 & 47 \\
\hline 7 & $406 \times 140 \times 39$ & $356 \times 127 \times 33$ & 1.60 & 0.64 & 1133.9 & 43 \\
\hline 8 & $406 \times 140 \times 39$ & $356 \times 127 \times 33$ & 1.80 & 0.56 & 1136.4 & 49 \\
\hline 9 & $406 \times 140 \times 39$ & $356 \times 127 \times 33$ & 1.60 & 0.64 & 1133.9 & 46 \\
\hline 10 & $406 \times 140 \times 39$ & $356 \times 127 \times 33$ & 1.45 & 0.83 & 1139.9 & 43 \\
\hline
\end{tabular}

However, varied depth necessitates building up an extra flange and web, and then welding them to the rafter which results in increased time and cost. It is recommended for haunch in eaves, besides using the same cross-section as rafter, to use the same depth as rafter. This is done in practice by cutting the section equal to the obtained optimum haunch length and halving it diagonally and longitudinally to avoid wasting the steel material. Having this idea, the optimum obtained weight was slightly heavier than using the varied depth. It increased the weight by $0.3 \%$ whereas the optimum haunch length was $2.40 \mathrm{~m}$. The convergence took place after 31 generation within 123 seconds time.

\subsubsection{Displacement maximisation}

In contrast to the weight minimisation, displacement maximisation does not assign consistent member sections to the rafters and columns. Furthermore, the optimum solution only happened once within ten runs. The reason refers to penalty function; as the violent individuals do not contribute in the crossover and mutation operations. The results show a bigger depth and smaller length of the haunch (Table 2) comparing to the weight minimisation. The optimum solution took place after 51 generation which has a weight of $1255.94 \mathrm{~kg}$. It is observed that the interaction limit reached to 1.00 whereas the lateral displacement did not reach the upper limit. 
In the case when the depth is fixed, the optimum solution is lighter than when a varied depth is the design variable. The weight reduced by $5 \%$ and the optimum solution reached after 49 generations with a haunch length of $0.70 \mathrm{~m}$ while the interaction limit reached to 0.99 and lateral displacement did not reach its upper limit.

Table 2: $\quad$ Result of lateral displacement maximisation.

\begin{tabular}{|c|c|c|c|c|c|c|}
\hline Run & $\begin{array}{c}\text { Column } \\
\text { section }\end{array}$ & $\begin{array}{c}\text { Rafter } \\
\text { section }\end{array}$ & $\begin{array}{c}\text { Haunch } \\
\text { length }\end{array}$ & $\begin{array}{c}\text { Haunch } \\
\text { depth }\end{array}$ & $\begin{array}{c}\text { Weight } \\
\text { Kg }\end{array}$ & $\begin{array}{c}\text { Gene- } \\
\text { ration }\end{array}$ \\
\hline 1 & $406 \times 178 \times 54$ & $305 \times 127 \times 48$ & 0.05 & 0.89 & 1518.2 & 39 \\
\hline 2 & $305 \times 165 \times 54$ & $305 \times 165 \times 54$ & 0.05 & 0.55 & 1635.5 & 52 \\
\hline 3 & $406 \times 178 \times 54$ & $305 \times 127 \times 48$ & 0.10 & 0.54 & 1520.2 & 54 \\
\hline 4 & $406 \times 140 \times 46$ & $356 \times 127 \times 39$ & 0.15 & 0.27 & 1255.9 & 51 \\
\hline 5 & $406 \times 178 \times 67$ & $305 \times 127 \times 42$ & 0.55 & 0.27 & 1540.3 & 48 \\
\hline 6 & $406 \times 140 \times 46$ & $305 \times 127 \times 48$ & 0.30 & 0.71 & 1455.8 & 42 \\
\hline 7 & $356 \times 171 \times 57$ & $305 \times 165 \times 46$ & 0.15 & 0.66 & 1512.0 & 53 \\
\hline 8 & $406 \times 178 \times 74$ & $305 \times 127 \times 42$ & 0.35 & 0.21 & 1602.0 & 56 \\
\hline 9 & $406 \times 178 \times 60$ & $305 \times 127 \times 42$ & 0.40 & 1.07 & 1484.4 & 53 \\
\hline 10 & $356 \times 171 \times 57$ & $356 \times 127 \times 39$ & 0.05 & 0.98 & 1364.3 & 54 \\
\hline
\end{tabular}

\subsection{Frame with dead and wind loads}

The second example is shown in fig 3. A combination of dead load with a value of $9.8 \mathrm{kN}$ generated from purlins and wind load with a value of $2.8 \mathrm{kN}$ are applied to the frame. Only one side of the frame is experienced by wind load whereas whole rafter of the frame is subject to the dead load. A notional horizontal force with a value of $0.049 \mathrm{kN}$ is applied to node 2 . The span of the frame is $24 \mathrm{~m}$ and the column and overall heights are $5.5 \mathrm{~m}$ and $7.0 \mathrm{~m}$ respectively. The same genetic parameters are used for this example.

\subsubsection{Weight minimisation}

The optimum length of the haunch has reached its upper range which is $6.40 \mathrm{~m}$ whereas the depth of haunch is quite small. The frame had a weight of $2557.6 \mathrm{~kg}$ at its optimum solution. $70 \%$ of the runs are the same section and haunch dimensions. The optimum solution was obtained after 46 generations. Unlike the displacements, the interaction limitation reached to its upper limit. The convergence lasted 156 seconds.

Taking the depth of haunch fixed, ended up with an $18 \%$ heavier frame while the interaction constraints control the design. The optimum obtained length was $1.60 \mathrm{~m}$.

\subsubsection{Displacement maximisation}

The obtained optimum design had an overall weight of $3315.1 \mathrm{~kg}$ which is $29 \%$ heavier than when the weight is minimised. In addition, the optimum solution does not take place consistently within 10 runs of the program. The convergence to optimum solution took place after 51 generations with last 231 seconds time. 
Once again, the interaction constraints control over the optimum design while the lateral displacements do not reach upper limit. However, the values of lateral displacements increased by $4 \%$ comparing to the weight minimisation.

Having the results considering fixed depth of the haunch, the optimum design comes up with slightly heavier weight than considering the varied depth by $0.4 \%$.

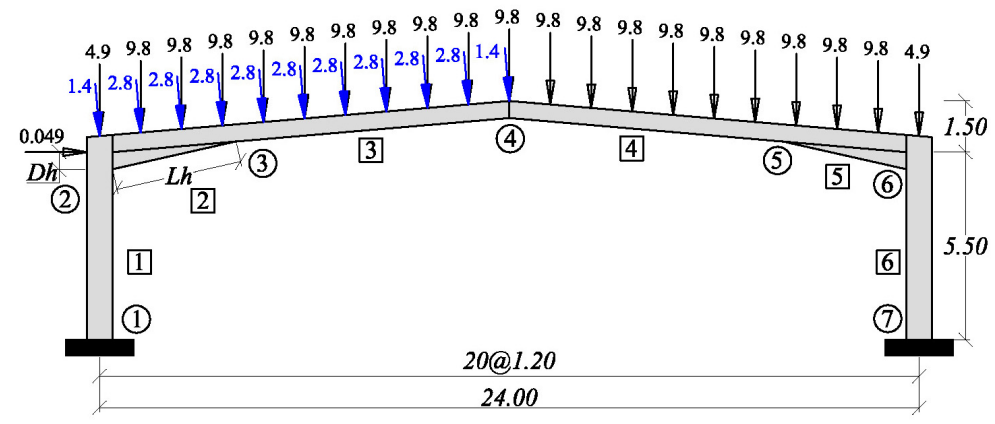

Notes:

All dimensions and loads are in ' $m$ ' and ' $k N$ ' successively.

Figure 3: Pitched-roof SPF experiences dead and wind loads.

\section{Conclusion}

A modified distributed genetic algorithm was used to enhance the speed of convergence into design solution. The modification includes using a varied mutation probability and bringing the elite individuals into the genetic operations twice while they are already secured to be dropped into the next generation. The mutation scheme assisted the algorithm to diversify the population in the earlier generation, due to high probability value, and making the design possible to access the feasible design space as much as possible. At later generations the probability value slipped down and allowed the problem to converge into the optimum solution, which happened within smaller number of generation, leading to a substantial decrease in computation time. The general stiffness matrix was another factor to reduce the computation time as it eliminated more loops for the integration for non-prismatic members. Using different mutation, reproduction schemes, fitness value calculation, DO-DGA can obtain the optimum solution within a few minutes so that it can be brought into daily office-use by structural engineers. Two different objective functions were used to appraise their efficiency. Two benchmark examples revealed that the minimisation of weight gives better results than maximisation lateral displacement does.

\section{References}

[1] Balling, R.J., Briggs, R.R., \& Gillman, K., Multiple optimum size/shape/topology designs for skeletal structures using a genetic 
algorithm. ASCE Journal of Structural Engineering, 132 (7), pp. 1158 1165, 2006.

[2] Camp, C., Pezeshk, S., \& Cao G., Optimum design of two-dimensional structures using a genetic algorithm. ASCE Journal of Structural Engineering, 124 (5), pp. 551 - 559, 1998.

[3] Degertekin, S.O., Saka, M.P., \& Hayalioglu, M.S., Optimal load and resistance factor design of geometrically nonlinear steel space frames via tabu search and genetic algorithm. Engineering Structures, 30, pp. 197-205, 2008.

[4] Foley, C., \& Schinler, D., Automated design of steel frames using advanced analysis and object-oriented evolutionary computation. ASCE Journal of Structural Engineering, 129 (5), pp. 648 - 660, 2003.

[5] Ghali, A., Neville, A.M., \& Brown, T.G., Structural Analysis; A Unified Classical and Matrix Approach, Spon Press: New York, 2003.

[6] Kameshki, E.S., \& Saka, M.P., Optimum design of non-linear steel frames with semi-rigid connection using a genetic algorithm. Computer and Structures, 79, pp. 1593-1604, 2001.

[7] Liu, X., Yi, W.J., Li, Q.S., Shen, P.S., Genetic evolutionary structural optimization. Journal of Constructional Steel Research, 5, pp. 346-358, 2007.

[8] Luo, Y.Z., Xu, X., \& Wu, F. Accurate stiffness matrix for non-prismatic members. ASCE, Journal of Structural Engineering, 133(8), pp. 11681175, 2007.

[9] Mühlenbein, H., Schomisch, M., \& Born, J., The parallel genetic algorithms as a function optimizer. Parallel Computing, 17, pp. 619-632, 1991.

[10] Pezeshk, S., Camp, C.V., \& Chen, D., Design of non-linear framed structures using genetic optimization. ASCE Journal of Structural Engineering, 126 (3), pp. 382 - 388, 2000.

[11] Saka, M.P., Optimum design of pitched roof steel frames with haunched rafter by genetic algorithms. Computers and Structures, 81, pp. $1967-$ 1978, 2003.

[12] Salter, P.R., Malik, A.S., \& King, C.M., ed., Design of single-span steel portal frames to BS 5950-1:2000. The steel Construction Institute: Berkshire, 2004.

[13] Toropov, V.V., \& Mahfouz, S.Y., Design optimization of structural steelwork using a genetic algorithm, FEM and a system of design rules. Engineering Computations, 18 (3/4), pp. 437-459, 2001. 